\title{
Frame-shifts of digit identity in bird evolution and Cyclopamine-treated wings
}

\author{
Alexander O. Vargas' ${ }^{1}$ and Günter P. Wagner* \\ Department of Ecology and Evolutionary Biology, Yale University, 165 Prospect Street, New Haven, CT, 06520-8106 USA \\ *Author for correspondence (email: gunter.wagner@yale.edu) \\ ${ }^{1}$ Present address: Departamento de Biología, Facultad de Ciencias, Universidad de Chile, Santiago, Chile.
}

\begin{abstract}
SUMMARY A highly conserved spatio-temporal pattern of cartilage formation reveals that the digits of the bird wing develop from positions that become digits 2, 3, and 4 in other amniotes. However, the morphology of the digits of early birds like Archaeopteryx corresponds to that of digits 1,2, and 3 of other archosaurs. A hypothesis is that a homeotic "frameshift" occurred, such that in the bird wing, digits 1, 2, and 3 develop from the embryological positions of digits 2, 3, and 4. Experimental homeotic transformations of single digits
\end{abstract}

are well-documented, but frame-shifts of more than one digit are not. We investigated the pattern of cartilage formation in the development of Cyclopamine-treated wings. When Cyclopamine was applied between stages 18 and 21, morphologies that normally develop from positions 2 and 3 developed from positions 3 and 4 . The serial shift of digit identity toward posterior confirms a mechanistic possibility that was previously inferred from the evolutionary history of birds.

\section{INTRODUCTION}

In pentadactyl amniotes there is a highly conserved spatiotemporal pattern by which the skeletal elements of the limb initiate cartilage formation. The first digital ray to initiate cartilage formation is always spatially in line with the ulnare and ulna, conforming a conceptual "primary axis" (red line in Fig. 1. Shubin and Alberch 1986) that develops into digit 4 (see Fig. 2). Digits 1, 2, and 3 develop in front of this axis (the "pre-axial" digits) whereas digit 5 develops posterior to it ("postaxial"). Birds evolved from pentadactyl reptiles; in the bird wing, the primary axis develops into the most posterior of the three definite digits (Fig. 1C). Because of this, the digits of the wing are considered to develop at embryological positions that become digits 2, 3, and 4 in other amniotes (Müller and Alberch 1990; Burke and Wagner 1997; Kundrát 2009). However, when we examine the evolutionary history of morphology in the lineage leading to birds, we observe that in early birds like Archaeopteryx, the three digits have a "phalangeal formula" $(2,3$, and 4 phalanges on each digit, respectively) that corresponds to that of digits 1,2 , and 3 of other archosaurs and reptiles (Romer 1950; Shapiro et al. 2007). Furthermore, the well-documented transition from early dinosaurs to birds reveals digits 4 and 5 became reduced and eventually lost, with only minor changes in digits 1, 2, and 3 (Gauthier 1986; Padian and Chiappe 1998).
Wagner and Gauthier (1999) proposed that, probably when digit 4 was lost, a "homeotic frame-shift" occurred, leading digits 1, 2, and 3 to develop from embryological positions that in other amniotes develop into digits 2, 3, and 4 (Fig. 1C). Since then, it has been shown that the absence of HoxD-10, HoxD-11, and HoxD-12 expression only in the anterior digit of the bird wing resembles that of digit 1 of other amniotes, suggesting that both morphology and Hox gene expression would have shifted simultaneously toward posterior (Vargas and Fallon 2005; Vargas et al. 2008).

Developmental research has often documented homeotic transformations of individual digits (Morgan et al. 1992; Knezevic et al. 1997; Dahn and Fallon 2000; Suzuki et al. 2004, 2008), but no serial, simultaneous "frame-shift" of digits like that proposed by Wagner and Gauthier (1999). Possible cases, such as the Shhc/Shh;MHoxCre mouse mutant (with reduced Shh expression; Lewis et al. 2001; Shapiro et al. 2003) are ambiguous because the pattern of cartilage formation has not been documented. Here we investigate cartilage formation in wings treated with Cyclopamine. Cyclopamine is a steroid alkaloid that decreases Shh signaling by binding to Smoothened protein, which is part of the hedgehog signaling cascade (Chen et al. 2002). By application of Cyclopamine to the wing bud at stages $18-21$, it is possible to produce the loss of the posterior digit that normally develops from the primary axis. No accompanying morphological changes 

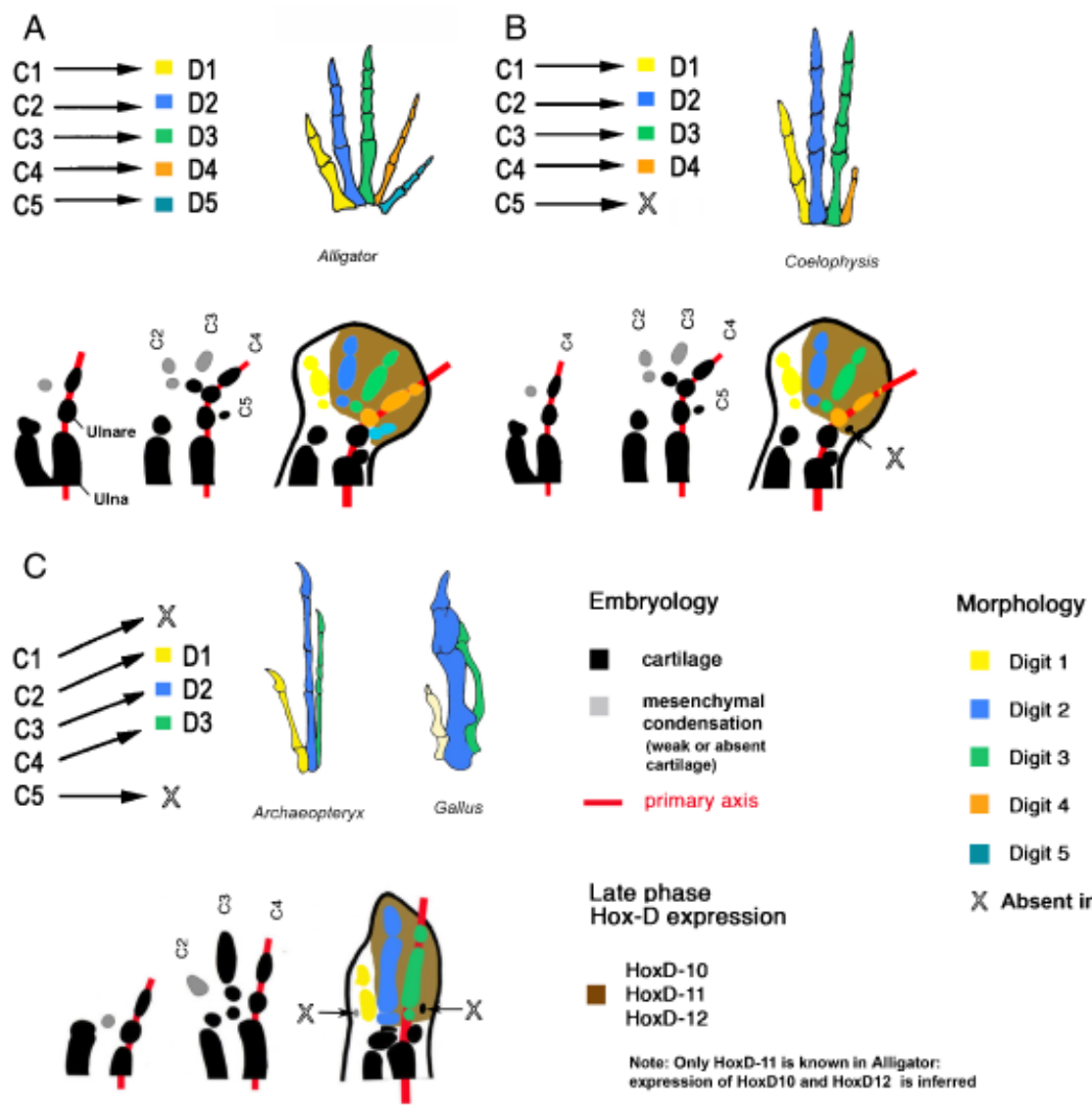

Fig. 1. The inference of a homeotic frame-shift in bird wing evolution. (A) The morphology and development in pentadactyl amniotes such as mouse and alligator. Cartilaginous digital condensations at different positions (C1-C5) develop into distinct adult digit morphologies (D1-D5, color coded). C4 is first to initiate cartilage formation and is spatially aligned with the ulnare and ulna, conforming the conceptual "primary axis" (indicated by the red line). HoxD-10, -11 , and -12 expressions are absent only at C1. (B) Loss of digit 5 in the early theropod ancestors of birds (as represented by Coelophysis). We propose that all digits D1-D4 developed as usual from condensations C1-C4, whereas C5 failed to develop into an adult digit (as in the bird foot, also missing only D5). (C) Loss of D4 in birds (and other Tetanuran theropods). In early birds like Archaeopteryx the three remaining digits had 2, 3, and 4 phalanges, clearly corresponding to D1, D2, and D3 of alligator and early theropods. However, the pattern of cartilage formation in birds indicates these digits develop from condensations $\mathrm{C} 2$, C3, and C4. Wagner and Gauthier (1999) proposed a homeotic frame-shift occurred such that in the wing C2, C3, and C4 develop into D1, D2, and D3. Vargas and Fallon (2005) pointed out that HoxD-10, -11, and -12 are also shifted, now absent only at C2 (D1). A mesenchymal condensation can be observed at the now "vacant" (unspecified) position of $\mathrm{C} 1$ ).

occur in the remaining anterior and middle digits (Scherz et al. 2007). This resembles the loss of the posterior digit 4 that occurred in the evolution of the bird wing, which did not affect the morphology of the remaining anterior digits (Fig. 1). Here we replicate these experiments to observe the pattern of cartilage formation as well as of HoxD-12 expression in the development of Cyclopamine-treated bidactyl (BD) wings. We found that the development of the anterior and middle digits, along with the anterior limit of HoxD-12 expression, is shifted one position closer to the primary axis. We conclude that a decrease in Shh signaling during a critical period of development can cause a shift in morphology and gene expression similar to that proposed for the evolution of bird wings.

\section{MATERIALS AND METHODS}

Eggs were incubated in polystyrene incubators (Hova-Bator Circulating Air model number 2362, G.Q.F. Mfg. Co. Inc., Savanna, CA, USA) at $39^{\circ}$ and windowed at day 3-3.5 to obtain embryos spanning stages 18-22. Hamburger-Hamilton stages were used for staging embryos at the moment of Cyclopamine application. Using a microinjector and glass needle, we delivered $5 \mu \mathrm{l}$ of $1 \mathrm{mg} / \mathrm{ml}$ solution of Cyclopamine (LC Laboratories, Woburn, MA, USA) in $45 \%$ 2-hydropropyl- $\beta$-cyclodextrin (HBC; Sigma, St. Louis, MO, USA) into the amniotic cavity, in direct contact with the embryo. To be delivered to the embryo, Cyclopamine must be first solubilized in 45\% HBC; Sigma. Like other authors, we found that $\mathrm{HBC}$ alone has no noticeable effects on the development of chicken limbs $(n=15 / 15)$. Whole-mount Alcian Blue staining and 
clearing of treated and control embryos at days 5, 6, and 10 using the technique described by Wassersug (1976). Whole mount in situ hybridizations were performed as previously described (Nieto et al. 1996). HoxD-12 probe is from Nelson et al. (1996).

\section{RESULTS}

\section{Replication of Cyclopamine experiments and resulting phenotypes}

To confirm that we could replicate the experimental results described by Scherz et al. (2007), we initially applied Cyclopa- mine aiming at a time-frame between stages 20 and 22 and observed the resulting phenotypes at day 10 or 11 of incubation. We obtained the same four phenotypes described by Scherz et al. (2007) within this time-frame; (A) Wings with a normal, "wildtype" (WT) phenotype (Fig. 2, A and F, 29\% $n=39 / 132$ ), (B) D2 fused to D3 (D2+D3; Fig. 2, B and F, $26 \% n=34 / 132$ ) (C) BD wing with D1 and D2 only (Fig. 2, C and $\mathrm{F}, 41 \% n=54 / 132)$. We also obtained a few specimens of a fourth severely reduced (SR) phenotype described by Scherz et al. 2007 that is extremely disorganized, with shortened humerus, loss of ulna, and two abnormal, stunted digits $(4 \%$
A

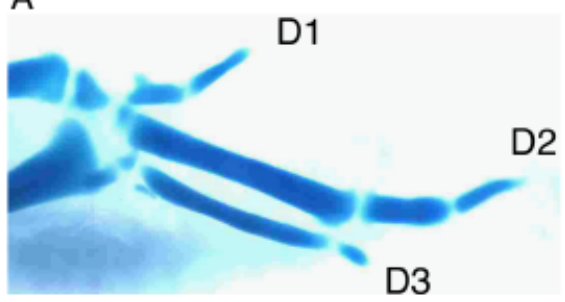

C

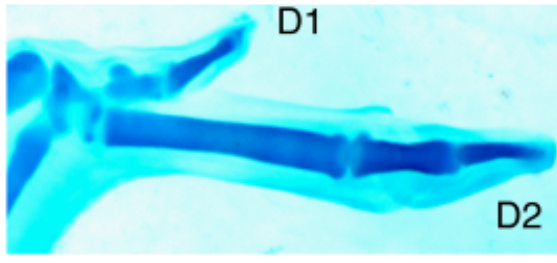

D2

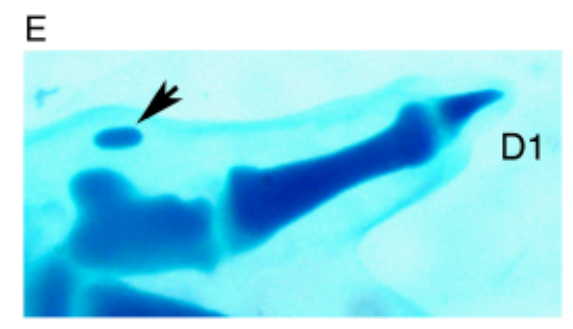

B

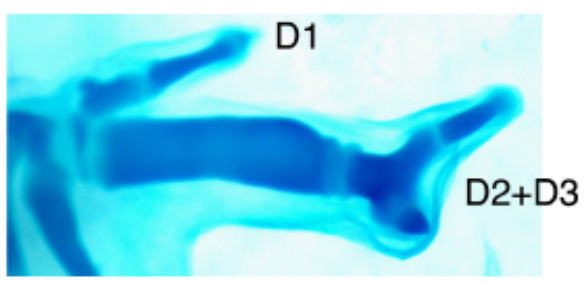

D

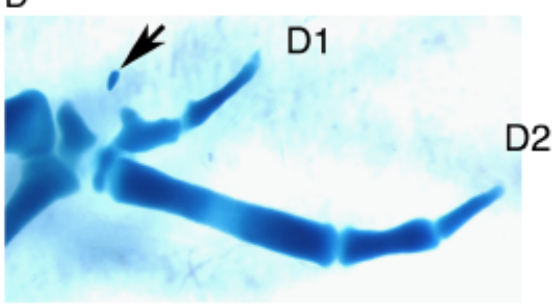

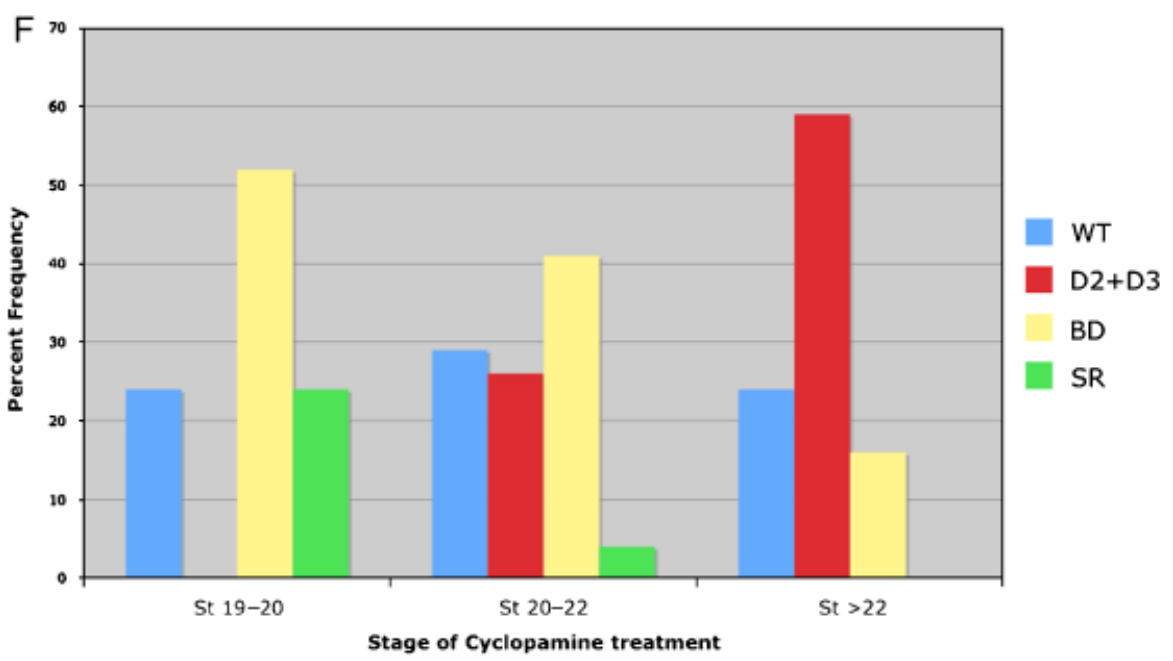

Fig. 2. Phenotypes after Cyclopamine treatment. Cyclopamine applied in a wide time frame (stages 20-23) can have the following outcomes at 10-11 days of incubation. (A) Wildtype phenotypes (WT, indistinguishable from control wings) are found. This phenotype is more frequent by application at stage 23 (Scherz et al. 2007), suggesting these embryos were injected too late. (B) Fused digits D2 and D3. D3 can also be reduced to varying degree. (C) Bidactyl phenotype; the posterior digit (D3) is lost; the remaining middle and anterior digits (D1 and D2) are morphologically unaltered, resembling the loss of D4 in bird evolution (see Fig. 1, B and C). (D) Bidactyl wing presenting the development of a cartilaginous nodule anterior to D1, at the position of $\mathrm{C} 2(n=9$ out of 64 bidactyl wings). (E) Close-up of the cartilaginous nodule anterior to D1 in another bidactyl specimen. (F) Frequency distribution of phenotypes in wings exposed at different stages of development. WT, wild type; $\mathrm{D} 2+\mathrm{D} 3$, fusion of digits 2 and 3; BD, bidactyle phenotype; SR, severely reduced wings. 
$n=5 / 132$, not shown). In the case of the $54 \mathrm{BD}$ wings obtained (phenotype C), nine of these specimens presented an ectopic cartilaginous element anterior to the first digit, at the same proximo-distal level as the digital condensations (Fig. 2, $\mathrm{D}$ and $\mathrm{E}$, black arrow). We believe our high number of $\mathrm{BD}$ specimens (54) allowed the detection of this phenotype, which was not reported by Scherz et al. (2007). In normal development, a vestigial cartilage (C5) is observed behind the primary axis, at the embryological position of digit 5 . This posterior cartilage was absent in all the altered phenotypes $(\mathrm{B}, \mathrm{C}$, and D). A few of the BD wings (7/54) had various degrees of ulna reduction: absent (no ulna), only proximal and/or distal ends, or a very thin, spike-like ulna. In one limb, the contour of a transparent ulna can be observed, suggesting the ulna is present but fails to produce cartilage ECM.

We also replicated the finer distinctions of time-frame sensitivity reported by Scherz et al. (2007). If Cyclopamine is applied at the later stages than 20-22, WT phenotypes and $2+3$ (fused) phenotypes are more frequent, and the SR phenotype is absent (WT $=24 \% 12 / 49,2+3=59 \% 29 / 49$, $\mathrm{BD}=16 \% 8 / 49)$ (Fig. 2F). When applied at stages 19-20, no phenotypes $2+3$ developed, the frequency of $\mathrm{BD}$ increased, and $\mathrm{SR}$ is now present $(\mathrm{A}=24 \% 7 / 29, \mathrm{C}=52 \% 15 / 29$, $\mathrm{D}=24 \% 7 / 29$ ). Our only noticeable difference with the results of Scherz et al. (2007) is that at all stages of injection we obtained a few WT wings. This could be due to occasional technical failure to effectively administer the Cyclopamine into the amniotic space.

\section{The pattern of cartilage formation in Cyclopamine- treated wings}

We targeted the early stages 19-20 for Cyclopamine treatment, that is, the stages which most consistently gave the $\mathrm{BD}$ phenotype, in order to observe the pattern of cartilage between days 5-6 (stages 27-30), which is when the primary axis and digit cartilages are formed in normal development. Observation at day 5 reveals some limbs that have not yet initiated the formation of cartilage in the autopodial segment $(n=5 / 20)$ that are indistinguishable from control wings at a comparable stage; there are also limbs where the first digital condensation is present in the primary axis and are indistinguishable from control limbs $(n=7 / 20)$ and wings that have a distinctly crescent-shaped posterior contour $(n=8 / 20)$ which we propose develop into abnormal phenotypes BD or SR. In all wings observed at day 5 (abnormal or not), the first digital condensation to initiate cartilage formation is formed spatially aligned with the ulna and the ulnare (Fig. 3A). We were unable to observe any limbs lacking the ulna at this stage; it is thus possible that the lack of ulna observed at later stages may relate to a disrupted maintenance of cartilage formation, rather than complete agenesis.

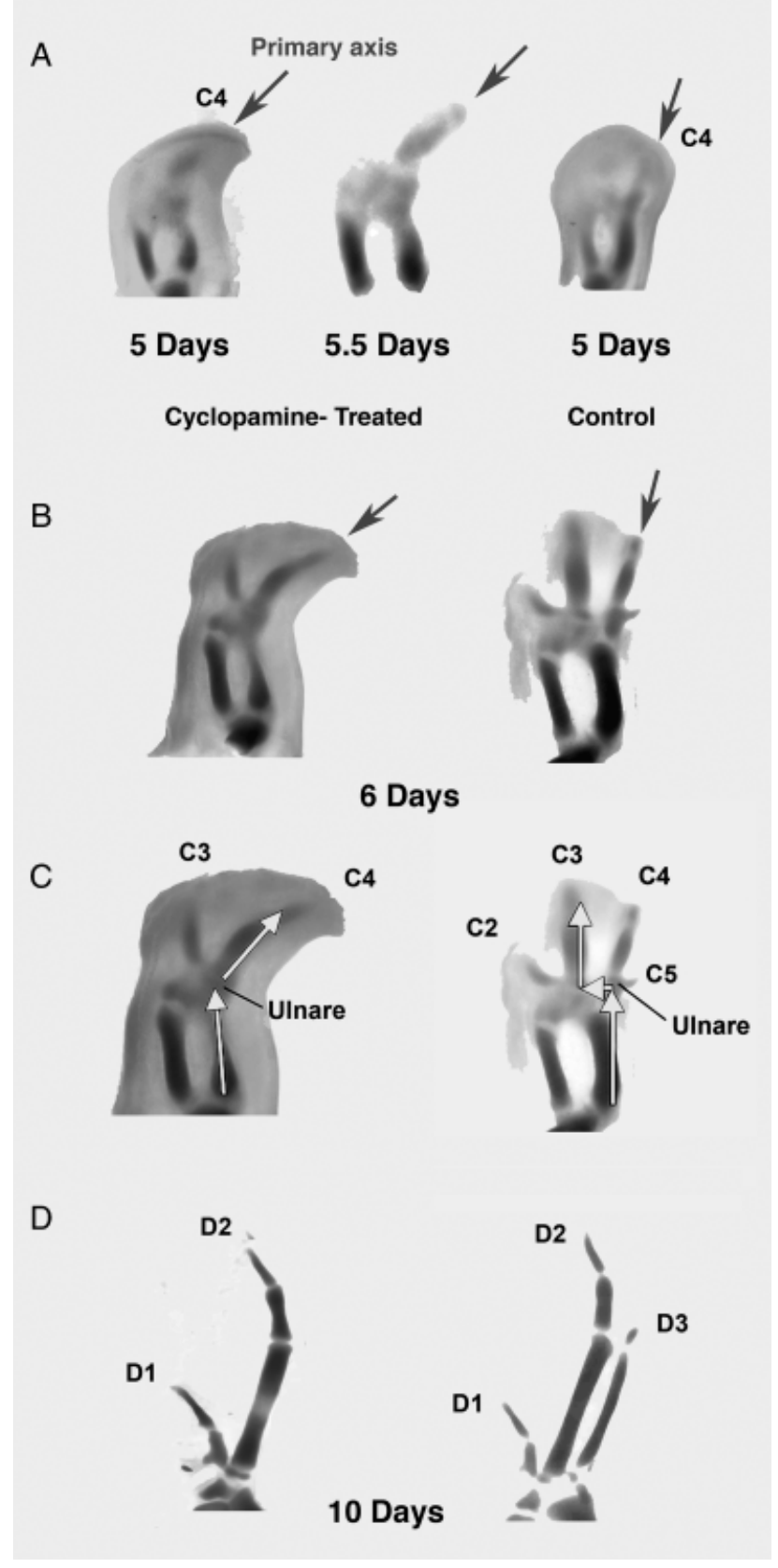

Fig. 3. The pattern of cartilage formation in Cyclopamine-treated wings. Cyclopamine was applied at stages 19-20. (A) At day 5 (stage 27-28) abnormal wings can be observed that have a crescent-shaped posterior contour. In both normal and abnormal wings, the first digit to initiate cartilage formation does so spatially aligned with the ulna and ulnare. (B) At day 6, wings that will develop into the bidactyl phenotype are evident. (C) The digits of bidactyl wings develop at the postion of condensations $\mathrm{C} 3$ and $\mathrm{C} 4$, with the second digit directly above the ulnare and ulna in bidactyl wings, one full position more toward posterior than in control wings (white arrows). (D) Bidactyl and normal phenotypes at day 10. C3 and C4 of bidactyl wings develop into D1 and D2, rather than D2 and D3 as in normal wings. 
At day 5.5 (stage 27-28), all wings have digit cartilages and we continue to observe control, normal phenotypes $(20 \%$, $n=12 / 60)$ and abnormal, BD phenotypes $(80 \%, n=48 / 60)$. As in day 5, most wings present an ulna that can be observed to be in line with the first digit to produce cartilage (Fig. 3A). Of the $48 \mathrm{BD}$ wings, 22 presented an abnormal ulna, which in most appears formed at the proximal and distal ends but presents lower or absent levels of cartilage formation at midlength. The developing autopod of four of these wings is smaller, suggesting they may correspond to the SR phenotype. At day 6 (stages 29-31), BD wings (69\% 43/62 Fig. 3B) can be confidently separated from WT wings (31\% 19/62). No SR wings were observed. Only 10 of the $43 \mathrm{BD}$ wings presented a reduced/absent ulna. In all wings presenting a well-developed ulna, it is evident that the second digit of the wing (D2) develops above the ulnare and the ulna (Fig. 3B), at the position of the primary axis that is usually occupied by the posterior digit (D3). In wings with a reduced ulna, the ulnare is still present at its normal posterior position, and D2 develops directly above it. We thus conclude that in Cyclopamine-treated $\mathrm{BD}$ wings, the second of the two remaining digits is developing from the embryological position $\mathrm{C} 4$, as indicated by its position directly above the ulna and ulnare. The final phenotype of this digit, however, is that of digit 2 . We consider this as evidence for a digit identity frame-shift induced by Cyclopamine.

\section{Expression of HoxD-12 in Cyclopamine-treated wings}

We observed the expression of the HoxD-12 gene at day 6 in Cyclopamine-treated $\mathrm{BD}$ wings. At this stage the phenotype of treated wings has become easily distinguishable. The position of HoxD-12 expression can be assessed by comparison with a red and stained WT and treated wings at the same stage (Fig. 4). HoxD-12 expression is absent at D1 in control $(n=6)$ and Cyclopamine-treated wings $(n=10)$. In Cyclopamine-treated $\mathrm{BD}$ wings, this corresponds to the embryological position of $\mathrm{C} 3$, rather than $\mathrm{C} 2$ as in control wing, confirming that both morphology and HoxD-12 expression are shifted toward posterior by one embryological position in Cyclopamine-treated wings.

\section{DISCUSSION}

\section{A frame-shift of digit identity toward posterior in Cyclopamine-treated wings}

At early stages of formation of the primary axis (day 5.5, Fig. $3 \mathrm{~A}$ ), all treated wings observed present a digital ray that forms in line with the ulnare and ulna. Because this includes the limbs that will develop into BD wings with high frequency, we

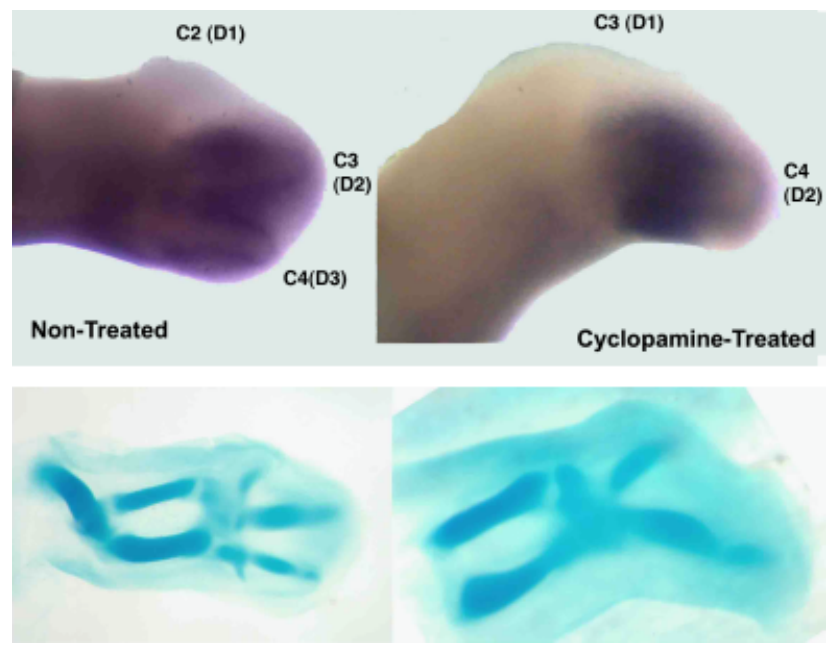

Fig. 4. HoxD-12 is shifted posterior in Cyclopamine treated as compared with control wings. Expression of HoxD-12 is presented at day 6. Cartilage preparations of wildtype and treated wings at the same stage are provided for reference. HoxD-12 expression is absent only in the most anterior digit of both control and Cyclopamine-treated wings. In Cyclopamine-treated bidactyl wings, the anterior limit of HoxD-12 is found one embryological position closer to the primary axis, resembling the shift of expression previously argued to have occurred in evolution (Vargas and Fallon 2005; Vargas et al. 2008).

must conclude that the primary axis in BD wings develops into the second digit (morphologically, digit 2) of Cyclopamine-treated wings. At a later stage (day 6, stage 29), limbs developing into $\mathrm{BD}$ wings are readily distinguishable; we observe that the longest digit, morphologically digit 2 , is directly above the ulnare rather than anterior to it (Fig. 3B, yellow arrows). Thus, we conclude that in the development of Cyclopamine-treated BD wings, the development of the anterior and middle digits has shifted toward posterior with respect to the position of the ulnare and ulna.

\section{The mechanism of posterior shift}

We hypothesize that, both in evolution and in shifts caused by Cyclopamine treatment, cells that remain in their spatial position become specified to more anterior identities. This can be achieved by altering Shh signaling (Riddle et al. 1993; Drossopoulou et al. 2000; Lewis et al. 2001). Shh is an important limb morphogen that is expressed in a posterior zone of the limb (the zone of polarizing activity [ZPA]); Shh protein is found in a diminishing gradient toward the more anterior digits (Riddle et al. 1993; Harfe et al. 2004). Thus, we assume that, by producing a generalized decrease in the levels of Shh signaling, Cyclopamine leads to the development of the anterior and middle digits (D1 and D2) at more posterior 

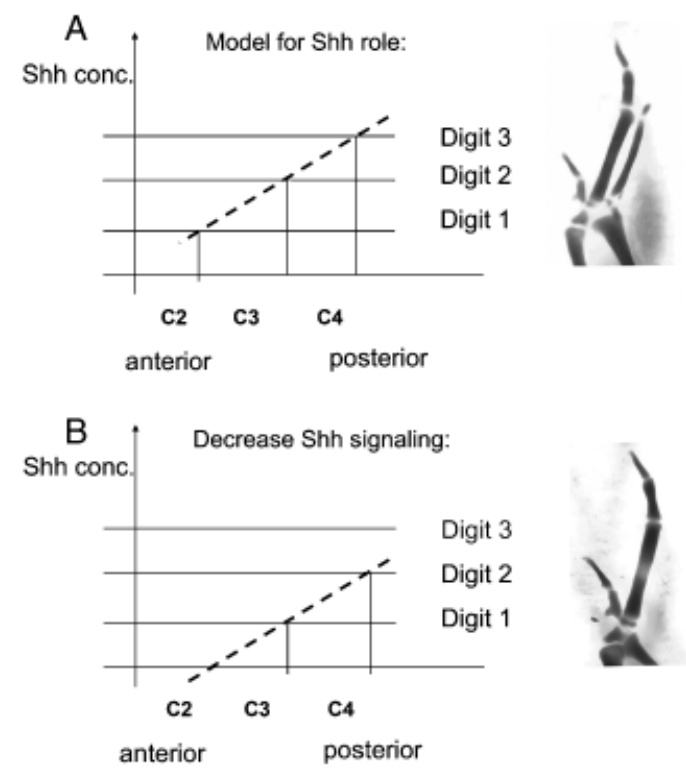

Fig. 5. A model of a Cyclopamine-induced identity frame shift. Shh protein in normal development is expressed in a posterior zone of the limb (the zone of polarising activity ZPA) from where it is found in a diminishing gradient toward anterior digital condensations. More anterior digital identities are specified by lower levels of Shh signaling. Cyclopamine binds to Smoothened, a downstream target of Shh, decreasing its signaling. We hypothesize that by producing a generalized decrease in the levels of Shh signaling, Cyclopamine leads to the development of more anterior digits (D1 and D2) at a more posterior position (C3 and C4).

positions (C3 and $\mathrm{C} 4$, Fig. 5). In 9/54 of BD wings, we observed a small cartilaginous nodule anterior of D1 (see Fig. 2). No such anterior cartilaginous nodule is ever found in normal chicken wings. This suggests that along with the posterior shift of identity, anterior cells with a potential for chondrogenesis at the embryological position of $\mathrm{C} 2$ are left with no specified digital identity, developing into a small "truncated" cartilage. The hypothesized homeotic frame-shift in evolution also implies that the most anterior embryological position (C1) was left "vacant," with no specified digital identity; in the ostrich, at this position, a corresponding anterior cartilage is apparent (Feduccia and Nowicki 2002). In the chicken, a small condensation exists that does not produce cartilage but can be detected by various other methods (Kundrát et al. 2002; Larsson and Wagner 2002; Van der Welten et al. 2005).

Although our study is focused on questions generated by previous analysis of evolutionary patterns, our data on cartilage formation suggests that posterior cells, which continue to produce Shh after Cyclopamine treatment (Scherz et al. 2007; Nissim et al. 2007), may contribute to the second digit (D2). If so, cells exposed to lowered levels of Shh signaling may develop anterior identities, regardless of whether they receive Shh signal or produce it themselves directly (Harfe et al. 2004).

\section{The parallel between evolution and Cyclopamine- induced digit identity shifts}

The hypothesis that a hometic frame-shift has occurred in the evolution of the bird wing has the following implications: (1) The correspondence of digital identity (adult morphology) with the pattern of cartilage formation has shifted, such that digits with more anterior identity develop in more posterior positions in the bird wing (Wagner and Gauthier 1999). (2) Along with morphology, HoxD-12 and HoxD-11 expression has also shifted one position toward posterior (Vargas and Fallon 2005; Vargas et al. 2008). (3) Anterior cells with potential to develop into a digit are left without specification; a vestigial condensation develops at this "vacant" position (Larsson and Wagner 2002). All of these previously inferred aspects were also found to be replicated by our experiments with Cyclopamine. This confirms that what was previously inferred as an event in the evolutionary history of birds is mechanistically possible through manipulation of Shh signaling. Because of the three levels of similarity, we propose a considerable overlap of the developmental pathways involved in both the evolutionary as well as the experimentally induced shift in digit identity.

\section{Acknowledgments}

This research was supported by NSF grant IOB-0445971 to G. P. W. and a Pew Latin American Fellowship to A. V. The authors thank Cliff Tabin, Harvard University, for sharing the then unpublished manuscript on Cyclopamine effects on limb development (Scherz et al. 2007) and for providing us with a clone of the chicken HoxD-12 gene for in situ hybridization.

\section{REFERENCES}

Burke, A. C., and Wagner, G. P. 1997. Developmental patterns and the identification of homologies in the Avian hand. Science 278: 666-668.

Chen, J. K., Taipale, J., Cooper, M. K., and Beachy, P. A. 2002. Inhibition of hedgehog signaling by direct binding of cyclopamine to smoothened. Genes Dev. 16: 2743-2748.

Dahn, R. D., and Fallon, J. F. 2000. Interdigital regulation of digit identity and homeotic transformation by modulated BMP signaling. Science 289: $438-441$.

Drossopoulou, G., et al. 2000. A model for anteroposterior patterning of the vertebrate limb based on sequential long- and short-range Shh signalling and Bmp signalling. Development 127: 1337-1348.

Feduccia, A., and Nowicki, J. 2002. The hand of birds revealed by early ostrich embryos. Naturwissenschaften 89: 391-393.

Gauthier, J. A. 1986. Saurischian monophyly and the origin of birds. Mem. Calif. Acad. Sci. 8: 1-55.

Harfe, B. D., Scherz, P. J., Nissim, S., Tian, H., McMahon, A. P., and Tabin, C. J. 2004. Evidence for an expansion-based temporal Shh gradient in specifying vertebrate digit identities. Cell 118: 517-528.

Knezevic, V., et al. 1997. HoxD-12 differentially affects preaxial and postaxial chondrogenic branches in the limb and regulates Sonic hedgehog in a positive feedback loop. Development 124: 4523-4536.

Kundrát, M. 2009. Primary chondrification foci in the wing basipodium of Struthio cemelus with comments on interpretation of autopodial elements in Crocodilia and Aves. J. Exp. Zool. (Mol. Dev. Evol.) 312B: $30-41$ 
Kundrát, M., Seichert, V., Russell, A. P., and Smetana Jr., K. 2002. Pentadactyl pattern of the avian wing autopodium and pyramid reduction hypothesis. J. Exp. Zool. (Mol. Dev. Evol.) 294B: $152-159$

Larsson, H. C., and Wagner, G. P. 2002. Pentadactyl ground state of the avian wing. J. Exp. Zool. (Molecular and Developmental Evolution) 294B: 146-151.

Lewis, P. M., et al. 2001. Cholesterol modification of sonic hedgehog is required for long-range signaling activity and effective modulation of signaling by Ptc1. Cell 105: 599-612.

Morgan, B. A., Izpisúa-Belmonte, J. C., Duboule, D., and Tabin, C. J. 1992. Targeted missexpression of Hox-4.6 in the avian limb bud causes apparent homeotic transformations. Nature 358: 236-239.

Müller, G. B., and Alberch, P. 1990. Ontogeny of the limb skeleton in alligator mississippiensis: developmental invariance and change in the evolution of archosaur limbs. J. Morphol. 203: 151-164.

Nelson, C. E., et al. 1996. Analysis of Hox gene expression in the chick limb bud. Development 122: 1449-1466.

Nieto, M. A., Patel, K., and Wilkinson, D. G. 1996. In situ analysis of chick embryos in whole mount and tissue sections. In M. Bronner-Fraser (ed.) Methods in Cell Biology. Vol. 51. Academic Press, New York, pp. 219 235.

Nissim, S., Allard, P., Bandyopadhyay, A., Harfe, B. D., and Tabin, C. J. 2007. Characterization of a novel ectodermal signaling center regulating Tbx2 and Shh in the vertebrate limb. Dev. Biol. 304: 9-21.

Padian, K., and Chiappe, L. 1998. The origin and early evolution of birds. Biol. Rev. 73: 1-42.

Riddle, R. D., Johnson, R. L., Laufer, E., and Tabin, C. J. 1993. Sonic hedgehog mediates the polarizing activity of the ZPA. Cell 75: 1401-1416.

Romer, A. S. 1950. The Vertebrate Body. 1st Ed. WB Saunders Company, Philadelphia.
Scherz, P. J., McGlinn, E., Nissim, S., and Tabin, C. J. 2007. Extended exposure to sonic hedgehog is required for patterning the posterior digits of the vertebrate limb. Dev. Biol. 308: 343-354.

Shapiro, M. D., Hanken, J., and y Rosenthal, N. 2003. Developmental basis of evolutionary digit loss in the Australian lizard Hemiergis. J. Exp. Zool. (Mol. Dev. Evol.) 297B: 48-56.

Shapiro, M. D., Shubin, N. H., and Downs, J. P. 2007. Limb diversity and digit reduction in reptilian evolution. In BK Hall (ed.). Fins into Limbs. The University of Chicago Press, Chicago, pp. 225-244.

Shubin, N. H., and Alberch, P. 1986. A morphogenetic approach to the origin and basic organisation of the tetrapod limb. Evol. Biol. 1: 319-387.

Suzuki, T., Hasso, S. M., and Fallon, J. F. 2008. Unique SMAD1/5/8 activity at the phalanx-forming region determines digit identity. Proc. Natl. Acad. Sci. USA 105: 4185-4190.

Suzuki, T., Takeuchi, J., Koshiba-Takeuchi, K., and Ogura, T. 2004. Tbx genes specify posterior digit identity through Shh and BMP signaling. Dev. Cell. 6: 43-53.

Van der Welten, M. C., Verbeek, F. J., Meijer, A. H., and Richardson, M. K. 2005. Gene expression and digit homology in the chicken embryo wing. Evol. Dev. 7: 18-28.

Vargas, A. O., and Fallon, J. F. 2005. Birds have dinosaur wings: the molecular evidence. J. Exp. Zool. (Mol. Dev. Evol.) 304B: 86-90.

Vargas, A. O., Kohlsdorf, T., Fallon, J. F., VandenBrooks, J., and Wagner, G. P. 2008. The evolution of HoxD-11 expression in the bird wing: insights from alligator mississippiensis. PLoS One 3: e3325. doi:10.1371 journal.pone. 0003325 .

Wagner, G. P., and Gauthier, J. A. 1999. 1,2,3=2,3,4: a solution to the problem of the homology of the digits in the avian hand. Proc. Natl. Acad. Sci. USA 96: 5111-5116.

Wassersug, R. 1976. A procedure for differential staining of cartilage and bone in whole formalin-fixed vertebrates. Stain Technol. 51: 131-134. 